\title{
Ulcerative Colitis complicated by lower limbs thromboemboilism- an unusual case scenario
}

\author{
Harpreet Singh, Rekha Mathur*, Parminder Kaur, Vikram Tanwar
}

Department of Medicine, P.G.I.M.S., Rohtak (124001), Haryana, India.

\section{DOI Name}

http://dx.doi.org/10.3126/jaim.v4i1.14178

\section{Keywords}

ulcerative colitis, arterial thrombosis, heparin, methyprednisolone, mesalamine

\section{Citation}

Harpreet Singh, Rekha Mathur*, Parminder Kaur, Vikram Tanwar. Ulcerative Colitis complicated by lower limbs thromboemboilism- an unusual case scenario. Journal of Advances in Internal Medicine 2015;04(01):28-30.

\begin{abstract}
Patients with inflammatory bowel disease (IBD) have an increased risk of vascular complications. In ulcerative colitis, $10 \%$ of deaths are attributed to thromboembolic complications. Arterial thromboembolic complications (ATEs) occur less frequently than venous thromboembolism (VTEs) in IBD patients. They are more common after interventional or surgical procedure but they can also occur spontaneously. Both venous and arterial, are serious extra-intestinal manifestations complicating the course of inflammatory bowel disease (IBD) and can lead to significant morbidity and mortality. Although there is no consensus regarding use of anticoagulants yet timely treatment of thrombosis in ulcerative colitis with anticoagulant therapy shows good results.
\end{abstract}

\section{INTRODUCTION}

Thromboembolic events, both venous and arterial, are rare and serious extra-intestinal manifestations complicating the course of inflammatory bowel disease (IBD). Arterial complications usually have embolic origins and represent rare but an important cause of mortality and morbidity in ulcerative colitis. The management of these conditions need multidisciplinary therapeutic approach and required awareness for prevention ${ }^{1}$. Here, a case of ulcerative colitis in a female, presented with thromboembolic event in arterial bed of right lower limb is being reported along with overview of literature.

\section{CASE REPORT}

A 40-year-old-female who presented with sudden onset blackish discoloration of right little toe which progressed to involve all the toes of right foot in 4 days (Fig 1). It was initially associated with pain followed by numbness in same region. For the last 7 years, patient had increased frequency of stools (>6 episodes/day) mixed with fresh red blood and mucus. Patient had history of low grade fever, decreased appetite. Vitals of the patient were stable. Patient had pallor, peripheral pulses examination revealed non palpable rt. dorsalis pedis and posterior tibial arteries. Painless, well demarcated blackish discoloration along with sensory loss appreciable on rt. mid dorsum, rt. mid planter \& rt. Forefoot (Fig 1). Rest of the systemic examination was normal.

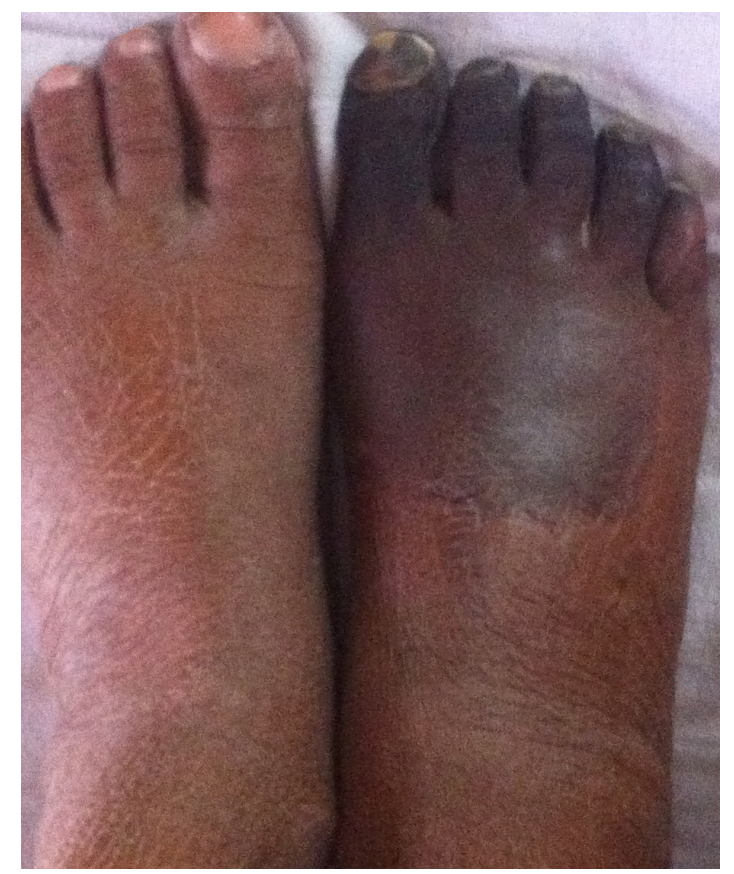

Figure 1 : Black discoloration of right foot in a patient with Ulcerative Colitis

\footnotetext{
* Corresponding author

Dr. Rekha Mathur

Department of Medicine, P.G.I.M.S.

Rohtak (124001), Haryana, India

Email: drrekhamathur04@gmail.com
} 
Laboratory investigations showed hemoglobin of $8.2 \mathrm{gm} / \mathrm{dl}$, total leucocyte count(TLC) of $8000 \mathrm{~mm}^{3}$, differential leucocyte count (DLC) of 78/18/02/02, platelets count of $6.8 \mathrm{lac} / \mathrm{mm}^{3}$ ESR $50 \mathrm{~mm} / 1^{\text {sthr, }}$. Microscopic examination of stool showed many pus cells and 6-8 RBC's. Coagulation profile showed prothrombin time (PT) 13.9 (control-13), activated partial thromboplastin time 28(control 28), Fibrin Degradation Product-positive. P-ANCA (MPO) 1.17 (<9.0). Viral serology for Human immune deficiency virus, Hepatitis-B Antigen and Anti-Hepatitis-C-Virus antibody were nonreactive. Echocardiography didn't document any thrombus.

On colonoscopy, multiple linear, serpiginous ulcers seen all over in transverse colon, descending colon, sigmoid colon, rectum, anal canal. Colonic biopsy showed variable acute inflammation, marked edema, mixed inflammatory infiltrate, minimal depletion of goblet cells and variable destruction of mucosa with extensive ulceration and cryptic abscesses which favored ulcerative colitis.

Color Doppler of right lower limb arteries: Popliteal artery showed biphasic flow with Peak Systolic Velocity (PSV) of 60$80 \mathrm{~cm} / \mathrm{sec}$, Anterior Tibial artery showed monophasic flow with PSV of $40 \mathrm{~cm} / \mathrm{sec}$ in proximal part and no flow could be demonstrated on distal half of leg, Posterior Tibial artery showed monophasic flow with PSV of $20 \mathrm{~cm} / \mathrm{sec}$ in proximal part and no flow detected in distal half of leg.

Contrast enhanced CT abdomen revealed a crescentric filling defect suggestive of atherosclerotic plaque along the posterior wall of abdominal aorta just proximal to the bifurcation of Aorta. CT angiography bilateral lower limbs showed reduced calibre of right profunda femoris artery. There was also reduced opacification of right anterior tibial and posterior tibial arteries (Fig 2,3).

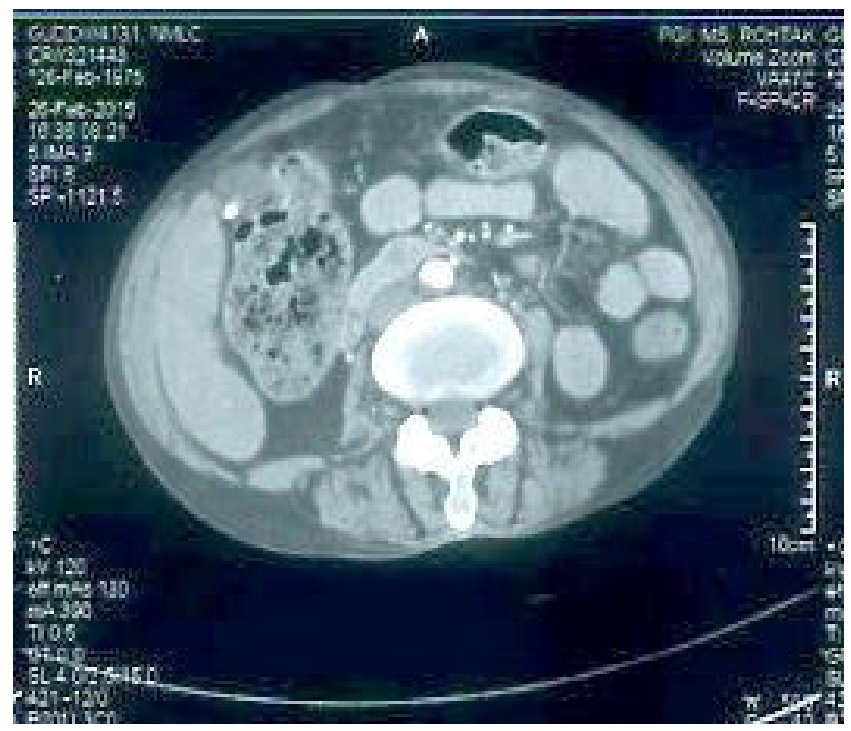

Figure 2: Contrast Enhanced CT abdomen showed cresentric filling defect in abdominal aorta

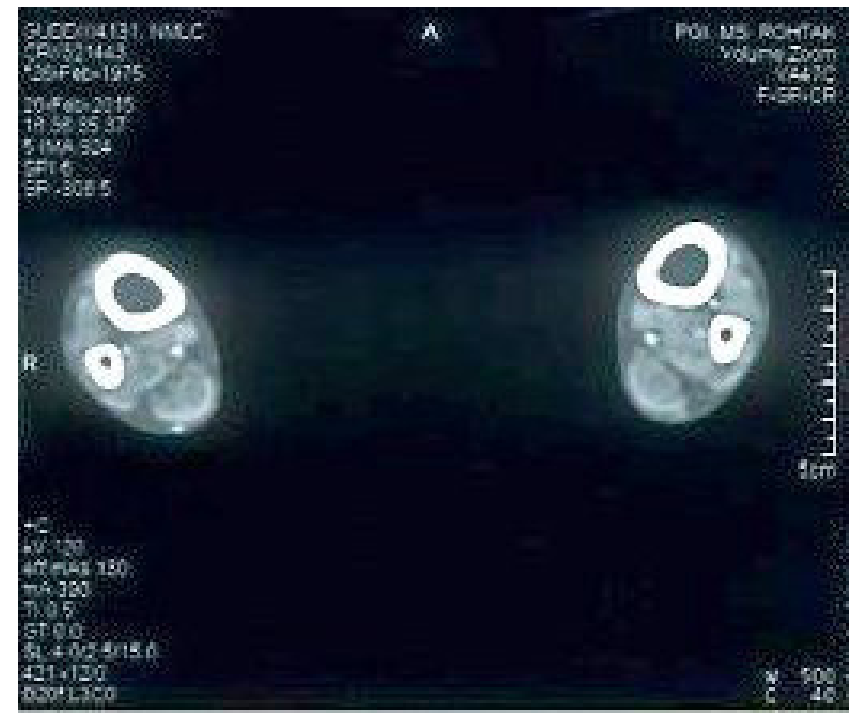

Figure 3: CT angiography bilateral lower limbs showed reduced calibre of right Profunda Femoris artery

Based on clinical profile and investigations, a diagnosis of idiopathic ulcerative colitis complicated by accelerated atherosclerosis with superimposed arterial thromboembolism was considered. Inj. Methylprednisolone $500 \mathrm{mg}$ IV for 3 days then shifted to tab. prednisolone $30 \mathrm{mg} O D$. along with tab. Mesalamine $2.4 \mathrm{~g}$ OD. After the confirmation of thromboemboism, she was started on subcutaneous enoxaparin 40mg twice a day and later switched over to warfarin after an overlap period of 5 days. After treatment, her stool frequency reduced to 3-4 episodes per day with semisolid consistency and there was no associated blood in stools. Sensations of fine touch, pain, vibration improved in the mid dorsal and mid planter aspect of right foot after 15 days.

\section{DISCUSSION}

Increased coagulability is an unusual but well-recognized phenomenon of ulcerative colitis. In 1936, Bargen and Barter $^{2}$ reported the association of thromboembolism complicating the course of IBD. Since then, several case reports have highlighted this rare association. An elevenyear study by Talbot showed that the thromboembolic complications occurred in $1.3 \%$ of patients with IBD of which peripheral arterial thrombosis had an incidence of less than 1 in 1000 patients with IBD ${ }^{3}$. Aortoiliac, femoropopliteal, and digital arteries are the most commonly involved vessels. ATEs occur less frequently and may involve the thrombosis and/or occlusion of the cerebral, retinal, carotid, coronary, splanchnic, iliac, renal, and limb (upper and lower) arteries or the aorta. It is more common in Crohn's disease than ulcerative colitis and generally occurs as a postoperative complication. Our case presented with dry gangrene of right foot with active disease 
not related to any operative complication.

In literature there are no specific coagulation defects ascertained to IBD have been found, although acquired deficiencies of antithrombin III and protein $\mathrm{S}$ have been reported. Although activated protein $C$ resistance is the most important cause of thrombosis in general population but its association with thrombosis in ulcerative colitis has not been found, but when present it increases the risk of thromboembolism. In literature, procoagulant activity in patients with active disease have shown elevation in levels of platelets, factors $\mathrm{V}$ and VIII, fibrinopeptides, and fibrinogen. ${ }^{4}$ The levels of these coagulation factors normalize with response to treatment of ulcerative colitis. ${ }^{4}$

Systemic effects of submucosal inflammation may have role in thrombotic events in IBD patients. It remains speculated whether mild or subclinical mucosal inflammation is capable of generating sufficient procoagulant activity to result in thromboembolic events. In ulcerative colitis, the majority of the patients do not have active disease at the time of thrombotic episodes unlike the present case, whereas the reverse is true in Crohn's disease. Other causes implicated in the causation/ accentuation of thrombosis include anemia, dehydration, and low cardiac output.

Treatment of thrombosis in patients with ulcerative colitis is not clearly defined in literature. The role of heparin in ulcerative

\section{REFERENCES}

1 Zitomersky NL, Verhave M, Trenor CC. Thrombosis and inflammatory bowel disease: a call for improved awareness and prevention. Inflamm Bowel Dis 2011;17(1):458-70.

2 Bargen JA, Barker NW. Extensive arterial and venous thrombosis complicating chronic ulcerative colitis. Arch Intern Med 1936;58:17-31.

3 Talbot RW, Heppell J, Dozois RR, et al. Vascular complications of inflammatory bowel disease. Mayo colitis is controversial. Anecdotal reports show the benefit of both unfractionated and low molecular weight heparin. ${ }^{4}$ It is not only the anticoagulant effect of heparin which is beneficial in thrombosis, it's probably the glycosaminoglycans constituent of heparin which has anti-inflammatory actions and to potentiate the activity of the peptide growth factors necessary for mucosal regeneration and repair so heparin has been suggested to have a possible role in refractory ulcerative colitis. ${ }^{4}$ Warfarin has not been found to have similar antiinflammatory role. ${ }^{4}$ However, a randomized, controlled trial reported an increase in significant bleeding complications with the use of heparin. ${ }^{5}$ Very limited data available on the use of either unfractionated or low-molecular-weight heparin for the treatment of ulcerative colitis ${ }^{6}$ but their use can be justified in those with associated thrombotic complications. Treatment with glucocorticoids affects blood coagulation, and a reduction of fibrinolytic activity may occur during long-term treatment, thus predisposing to venous thrombosis. Drug interaction occurs due to concomitant sulfasalazine and azathioprine, for ulcerative colitis with warfarin resistance requiring significant increases in warfarin dosage. This case highlights the tendency for thrombosis/thromoembolism in ulcerative colitis which may or may not be related to activity of disease and the need for vigilant approach for early recognition of these rare but dreadful complications occurring during course of ulcerative colitis and appropriate management of these complications.

\section{Clin Proc 1986;61:140-5.}

4 Wakefield A, Cohen Z, Levy G. Procoagulant activity in gastroenterology. Gut 1990;31:239-41.

5 Srivastava AK, Khanna N, Sardana V, et al. Cerebral venous thrombosis in ulcerative colitis. Neurol India 2002;50:215-7.

6 Panes J, Esteve $\mathrm{M}$, Cabre $\mathrm{E}$, et al. Comparison of heparin and steroids in the treatment of moderate and severe ulcerative colitis. Gastroenterology 2000;119:903-8. 